\title{
Application of Radical Basis Function Neural Network in Fault Diagnosis of Rectifier
}

\author{
DONG Jin-zhao ${ }^{1}$, XIE Yong-cheng ${ }^{2}$, HUANG Jie-yin ${ }^{1}$, ZHAI Ying-wen ${ }^{1}$ \\ ${ }^{1}$ Department of Control Engineering, Academy of Armored Forced Engineering, Beijing, 100072, China \\ ${ }^{2}$ Department of Foreign Training, Academy of Armored Forced Engineering, Beijing, 100072, China \\ langzixy@qq.com,15210080475
}

\begin{abstract}
The Radical Basis Function (RBF) neural network is a kind of three-forward neural network, which can approximate any continuous functions to arbitrary precision, particularly suited to solve classification problems. In this paper, according armored vehicle power system silicon rectifier prophase fault is single diode and diode short-circuit fault in a very short period of time turned into the situation of the open-circuit fault, make full use of the characteristics of the RBF network classification to determine the fault to a special diode of the rectifier model. It achieves the aim at fault diagnosis of rectifier. Comparing with BP neural network, RBF neural network has better classification ability.

Index Terms - RBF neural network, Rectifier, Fault diagnosis.
\end{abstract}

\section{Introduction}

At present, army armored vehicles mostly adopt brushless silicon rectifying generator, which consists of a three-phase ac low voltage generator and an air-cooled three-phase bridge rectifier with silicon rectifier diode. Among them, the threephase alternating current coming from the generator rectifier outputs into direct current to supply the power device and generator self excitation, and also for battery charging. As the constant voltage power supply for the whole vehicle electrical and electronic equipments, the rectifier has played an important role in guaranteeing the reliability and quality of the power system. Therefore, it has important practical application value to carry out fault diagnosis method research on rectifier. There are many literatures for the rectifier device fault diagnosis is studied. Among them, the literature [2] is proposed based on support vector machine (SVM) fault diagnosis method of rotating Rectifier Bridge, literature [3] is proposed a rectifier based on SOM and ELMAN neural network fault diagnosis methods, literature [4] puts forward a kind of fault diagnosis methods based on artificial immune algorithm brushless excitation generator. They all obtain a good diagnosis effect from the simulation results.

RBF neural network belongs to the type of feed forward neural networks, in which the underlying layer uses nonlinear optimization strategy to adjust the parameters of the activation function. And the output layer adopts linear optimization strategy of the linear power adjustment. It can approximate any continuous function with arbitrary precision, especially suitable for solving the problem of classification. Overall, the network mapping from input to output is nonlinear, but the network output for adjustable parameters are linear. The adjustable parameters of the network can work out directly by the linear equation, which greatly accelerates the learning speed and avoids local minimum problem [5]. This paper takes full advantage of the characteristics of RBF neural network and flexibly uses in the rectifier fault diagnosis. It is of great significance for intelligent fault diagnosis.

\section{II . RBF Neural Network [5]}

The neuron structure of Radical Basis Function (RBF) neural network is shown in figure 1 . The basis function of the activation function uses radial basis function, which usually defined as the space of Euclidean distance between any points into the center of a monotonic function.

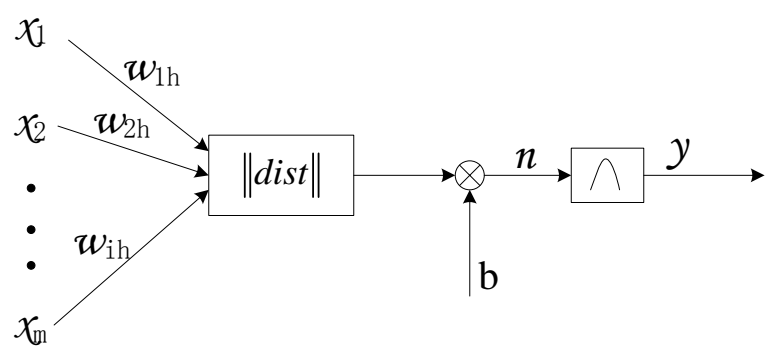

Figure 1 radial basis neural model

From figure 1 you can see that the radial basis neural network's activation function is based on the distance between the input vector and weight vector as independent variables. The general expressions of RBF neural network's activation function is:

$$
R(\|d i s t\|)=e^{-\|d i s t\|^{2}}
$$

As the decrease of the distance between the input vector and weight, the network output is increasing. When the input vector and weight vector is consistent, neurons output 1 . In the figure $1 \mathrm{~b}$ is for the valve value, in order to adjust the sensitivity of neurons. Using RBF neuron and competition neurons can form a probability neural network; this network is applicable to solve classification problems.

Consists of input layer, hidden layer and output layer general RBF neural network structure is shown in figure 2. 


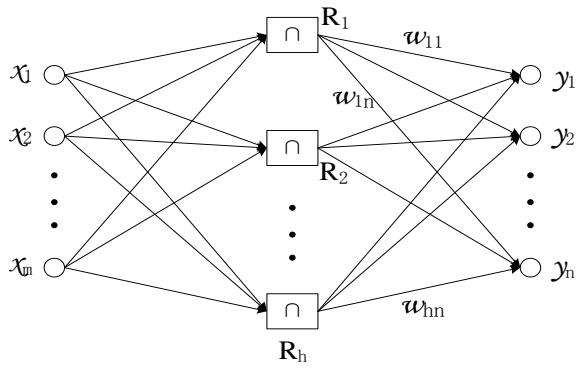

Figure 2 RBF neural network structure

In RBF network, input layer only has the effect of transmission signal, hidden layer is used to adjust the parameters of the activation function by nonlinear optimization strategy, and the output layer adopts linear optimization strategy for linear power adjustment. Thus it can approximate any continuous function to arbitrary precision. RBF neural network learning algorithm has 3 parameters to solve: the center of the basis function, variance and weights of hidden layer to output layer. As commonly used in radial basis neural network of radial basis function is Gaussian function, so RBF neural network's activation function can be expressed as

$$
R\left(x_{p}-c_{i}\right)=\exp \left(-\frac{1}{2 \sigma^{2}}\left\|x_{p}-c_{i}\right\|^{2}\right)
$$

$\left\|x_{p}-c_{i}\right\| \longrightarrow$ Euclidean norm

$c$ _ center of the Gaussian function

$\sigma \quad$ the variance of Gaussian function

From figure 2 radial basis neural network structure can obtain the output of the network as follows:

$$
y_{j}=\sum_{i=1}^{h} w_{i j} \exp \left(-\frac{1}{2 \sigma^{2}}\left\|x_{p}-c_{i}\right\|^{2}\right), j=1,2, \cdots, n
$$

$x_{p}=\left(x_{1}^{p}, x_{12}^{p}, \cdots, x_{m}^{p}\right)^{T} \longrightarrow$ The P-th input sample

$c_{i}$ center of the network hidden layer nodes

$w_{i j}$ the weights between hidden layer to output layer

$i=1,2, \cdots, h \_$the hidden layer nodes.

$y_{j}-$ actual output of $\mathrm{j}$ Output node

Assume that $d$ is the desired output of samples; Variance of basic functions can be expressed as

$$
\sigma=\frac{1}{P} \sum_{j}^{m}\left\|d_{j}-y_{j} c_{i}\right\|^{2}
$$

\section{III . Establishment of Model System}

The armored vehicle power supply system of the rectifier all adopt three-phase bridge uncontrolled rectifier circuit. The literature [6] gets a good simulation effect by MATLAB/Simulink. It provides a schematic diagram shown in figure 3.

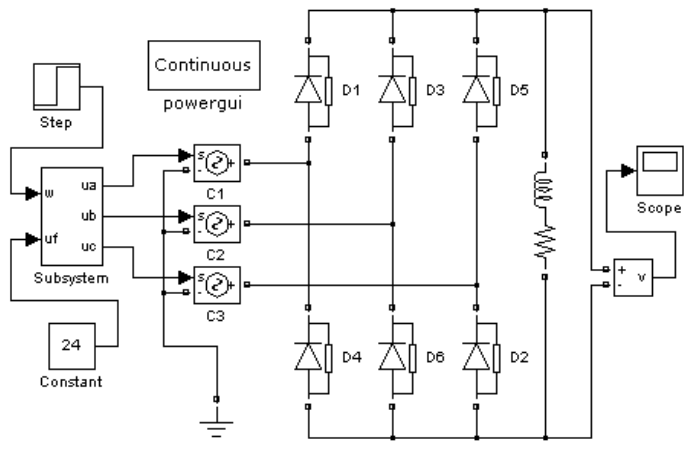

Figure 3 the schematic diagram of rectifier

Subsystem is the encapsulated module of generator mathematical model. Due to the connection between signal port and the power port cannot be directly connected; the controlled voltage source is adopted to improve the conversion.

\section{Fault Classification Design}

Rectifier is an important element in ac synchronous generator excitation system. As frequently used in high speed rotating state, and be affected by over-voltage, over current and other abnormal factors, rectifier diode element may occur open circuit or short circuit faults [7-9]. Through the literature, the present method of fault diagnosis of rotating rectifier mainly concentrated in the harmonic component analysis of exciter excitation current. But the conclusions are not consistent, and most of them are qualitative description. In addition, the rotating rectifier faults are divided into diode short circuit and open circuit fault. In actual operation, the diode will be converted into open circuit fault in a short amount of time. And considering the fault is usual a tube fault [10], so this paper will focus on tube faults of rotating rectifier diode. The rectifier faults can be divided into 7 kinds: normal working state; D1 open circuit; D2 open circuit; D3 open circuit; D4 open circuit; D5 open circuit; D6 open circuit. Because each fault waveforms are basic unanimous, simulation results are not listed one by one. As waveforms are obviously different in phase, then select "trouble-free", D1 and D4 fault simulation results showing in figure 4.

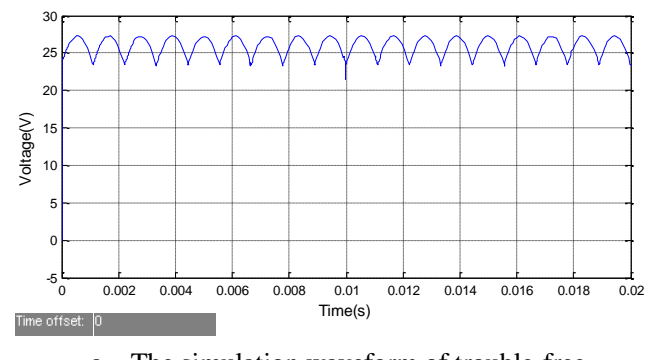

a The simulation waveform of trouble-free 


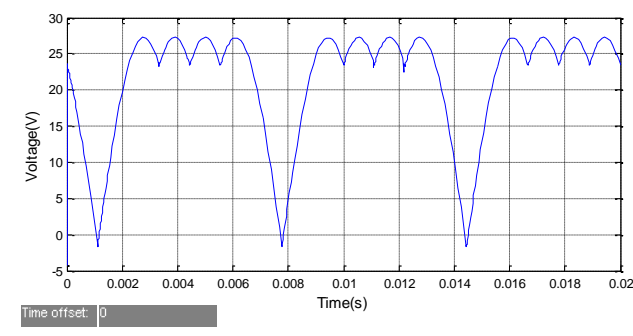

b V The simulation waveform of D1 open circuit

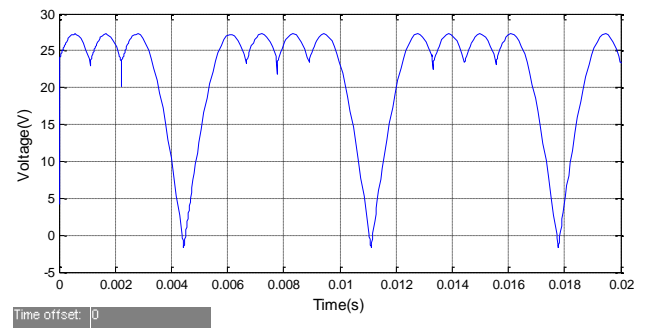

c 、 The simulation waveform of D4 open circuit

Figure 4 simulation waveform of single pipe rectifier open-circuit fault

Therefore, MATLAB programming can be used for various waveform sampling. Then use RBF neural network to data processing, determine the specific diode fault through comparing the network training output diagnosis results and desired output. Types of expectations in system various faults are shown in table 1.

Table 1 expectations of fault

\begin{tabular}{|l|l|l|l|}
\hline expectation & fault & expectation & fault \\
\hline 1000000 & None & 0000100 & D4 \\
\hline 0100000 & D1 & 0000010 & D5 \\
\hline 0010000 & D2 & 0000001 & D6 \\
\hline 0001000 & D3 & & \\
\hline
\end{tabular}

In the guarantee from the sampling points can accurately reflect the characteristics of waveform and not increasing the complexity of the network, 50 sampling points are set per cycle. Take two cycles as example. So there are 100 points in each fault type. Set the sampling interval In the Scope parameters. Then select the Save data to the workspace. Then set the Format type as Array. Then run the Simulink, program it as a RBF neural network input vector.

\section{The Training and Validation of the Network}

Write neural network training program In the MATLAB $M$ file. In the process of programming, the most important parameter is distributed constant of the radial basis function (RBF).As the sample data are

Due to large sample data, to set the distribution of the constant spread of 1.5.Using radial basis function in matlab neural network toolbox. The function can automatically add the number of hidden layer neurons, until the mean square errors meet the requirements. So the network creation process is the training process.

In order to verify the correctness of this network, some tests on samples are needed. After running $\mathrm{M}$ file, diagnosis of test samples can be found within the workspace. Comparisons are shown in table 2 .

Table 2 comparison of RBF network output vector and the desired output vector

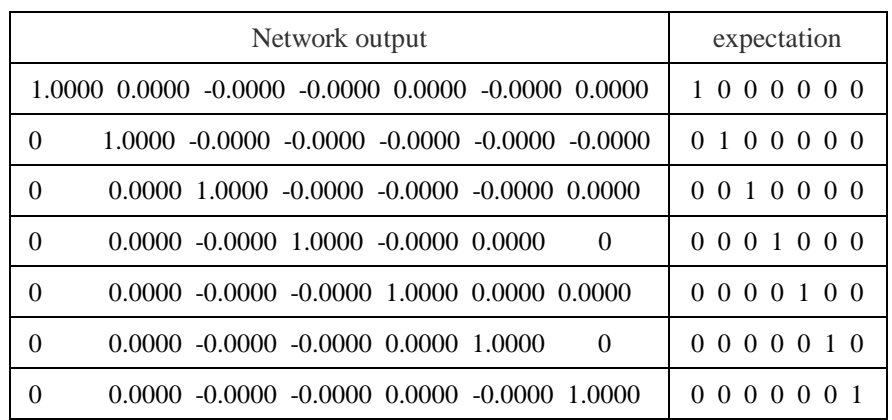

As it can be seen from the table, the output of the network vector and the desired output vector is consistent, so as to verify the validity of the network, at the same time also shows that the network has strong ability of classification.

In order to carry out comparison with the method proposed in this paper, the same sample is used as input vector of BP neural network. The network node number of input layer neurons is: $\mathrm{N}=100$. The network node number of output layer node number is: $M=7$. Node number of implicit layer can be referenced to American scientists Hebb proposed empirical formulas:

$$
h=\sqrt{N+M}+a
$$

The " $\mathrm{a}$ " is a constant one to ten.

The " $\mathrm{h}$ " value range is 12 to 20 . After many experiments, select "h" as17. Hidden layer neuron selects function tensing [11] (s-shaped tangent function) as activation function. The output layer neurons [11] chooses losing function as activation function. Set training times 2000 and training objective net.trainParam.goal $=0.01$. The network training error curve is shown in figure 5:

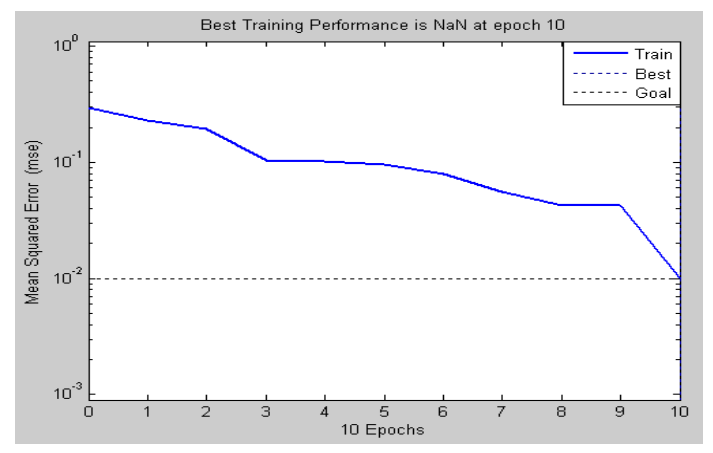

Figure 5 Error convergence diagram 
Its network diagnostic output and desired output vector are compared in table 3

Table 3 BP network output vector and the desired output vector

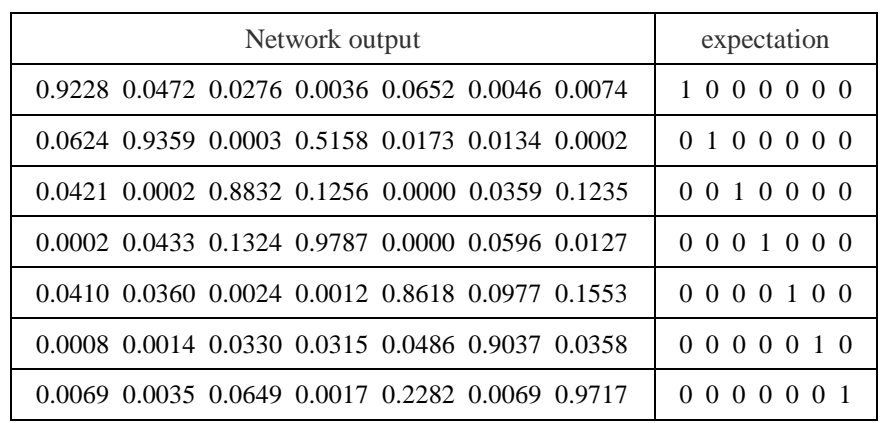

By comparing the table 2 and table 3, RBF network of training results is superior to the BP neural network of training results.

\section{Conclusion}

In this paper, according the armored vehicle power system silicon rectifier prophase fault is single diode and diode shortcircuit fault in a very short period of time turned into the situation of the open-circuit fault, analyzed the fault diagnosis method of single diode open circuit. Take each diode fault waveform sampling data as the input vector and use RBF neural network for network training. The result shows that classification of this method is better than the BP neural network algorithm. It can solve the problem of a single diode fault classification well, avoid removing the generator for inspection one by one. This method saves the time of fault diagnosis and achieves the purpose of the rectifier fault diagnosis. At the same time, this method can also be extended to other research and application of intelligent fault diagnosis.

\section{Acknowledgment}

In this paper, I am grateful for the guidance of my dear teacher Xie Yong-cheng, also thanks to the hard work fellow Huang Jie-yin.

\section{References}

[1] Y.N Zhang, Y.C Xie, Armored Vehicle Electrical and Electronic Systems, Beijing: National Defense Industry Press, 2003.

[2] Y.W Cheng, Y.C Xie, G.S Li, Fault diagnosis of rotating rectifier bridge based on SVM, Computer Measurement \& Control, vol. 19, no. 3, pp. 516-518, March 2011.

[3] H.M Kang, Study on design for testability and fault diagnosis of armored vehicle's power system, China: Academy of Armored Forces Engineering, 2012.

[4] N. Liu, C. Xie, et al, Immune algorithm in rotating rectifier fault diagnosis of brushless generator, Electric Power Automation Equipment, vol. 27, no. 5, pp. 32-35, May 2007.

[5] D.F Zhang, MATLAB Neural Network Application Design, Beijing: China Machine Press, 2009.

[6] G.S Li, H.M Kang, Y.C Xie, N. Wei, Research on fault diagnosis method of armored vehicle power system based on BP neural network, Computer Measurement \& Control, vol. 20, no.10, pp. 2719-2721, October 2012.

[7] C. Zhang, L. Xia, Fault diagnosis of generator rotating rectifier based on fractal and dynamics, Electric Machines and Control, vol. 13, no. 1, pp. 6-10, January 2009.

[8] McArdle M G, Morrow D J. Noninvasive Detection of Brushless Exciter Rotating Diode Failure, IEEE Transaction on Energy Conversion, vol. 19, pp. 378-383, February 2004.

[9] Y.Z Liu, C. Liu, Fault diagnose of rotating rectifier based on EMD and LS-SVM, vol. 45, no. 4, pp. 21-24, April 2012.

[10] C. Zhang, L. Xia, Reviews on fault diagnosis for AC brushless generators, vol.21, no.9, pp.103-106, September 2007.

[11] D.F Zhang, MATLAB/Simulink Modeling and Simulation Instance Succinctly, Beijing: China Machine Press, 2010 